\title{
Future of Social Network with Collaboration of Cloud Computing and Brain Computer Interface
}

\author{
Sraboni Barua \\ Lecturer, Dept. of CSE \\ Dhaka International University
}

\author{
Tamim Al Mahmud \\ Lecturer, Dept. of CSE \\ Dhaka International University
}

\author{
Samrat Kumar Dey \\ Lecturer, Dept. of CSE \\ Dhaka International University
}

\author{
Md. Motiur Rahman \\ Lecturer, Dept. of CSE \\ Dhaka International University
}

\begin{abstract}
In current time, people are greatly depending on social network for many purposes like communicating, advertising, knowledge sharing etc. The needs are so high that people sometime need to stay logged in for all time. But people have other works too and it is hardly to concentrate on another work while chatting with a friend. We made a survey to get the real scenario of Facebook uses and discovered how it decreases working efficiency. Focusing on the problem a future technology based solution is proposed. The solution emphasized on brain computer interfacing (BCI) to access the social networks. Implementing nanotechnology and photonic communication technology provides the portability of the system for all time as well as less power consuming. A cloud computing platform is used for data resource and to store other necessary commands. For processing massive data in the cloud platform a map reducing formula is described shortly. The whole system acts as a single process in the human brain and gives the ability to do another work at the same time.
\end{abstract}

\section{General Terms}

Human Brain Interfacing, Cloud Computing, Future Social Network

\section{Keywords}

Social network, Brain computer interface, Cloud computing, Nano technology, Mat lab to map reduce.

\section{INTRODUCTION}

Social media is the great revolution of the decade. Days are rapidly changing due to the change in communication systems. People all around are trying to cope up with the change. Hence they have to be connected and use social networks. Simply these social networks are looking useful though there are some harmful effects as well. It may impact on working performance of daily life. Firstly, the study gives a survey result on average uses time of Facebook per day. The output is not so negligible and it requires the next step to know either it hampers people's working efficiency or not. It shows the need to develop a future system for social networking. The future system is nothing but a combination of BCI and cloud computing. BCI describes the interfacing between brain and device. Cloud serves the resource and data. Using Nano technology [1], the system can be embedded into the human brain. It takes a small electric signal from neurons and executes proper command. The system works with a very small part of the brain and acts like a single process. So using this person can connect to the social networks for all time of the day without reducing his working efficiency.

\section{SURVEY ON FACEBOOK USEAGE AT CURRENT TIME}

As an early study, we made a manual survey on 100 Facebook users and found some unusual information.

\subsection{Scenario of Facebook Uses Time a Day}

A big amount of time people uses to stay in touch of Facebook, twitter etc. Firstly, people were asked that how many hours they stay in Facebook a day. Most of the people $(40 \%)$ uses Facebook minimum two hours a day and a big number of people stay connected more than five hours (Figure 1). Question is if people cost their five hour from 24 hours of the day to use social network, then their working efficiency is destroying or not. On the other hand, the need of the social network cannot be denied and it would be better if people can connect all time in the social media without hampering daily work.

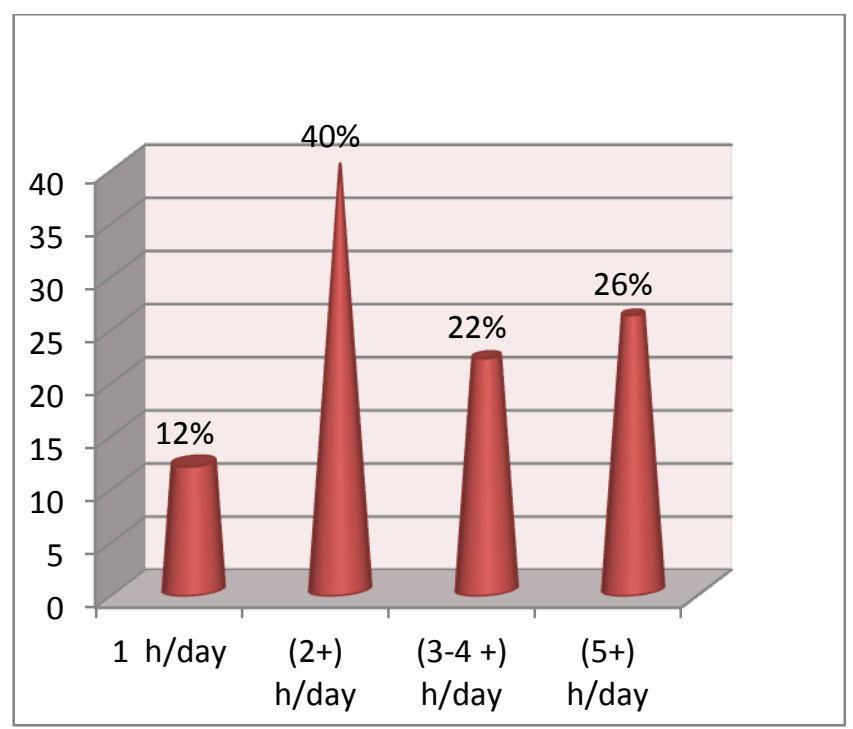

Fig 1: Hourly uses time of Facebook per day. 


\subsection{Current System Decreases Working Efficiency}

The next question is to the students and its result is little horrible.72\% students often uses Facebook in their classroom by mobile (Figure 2 ).

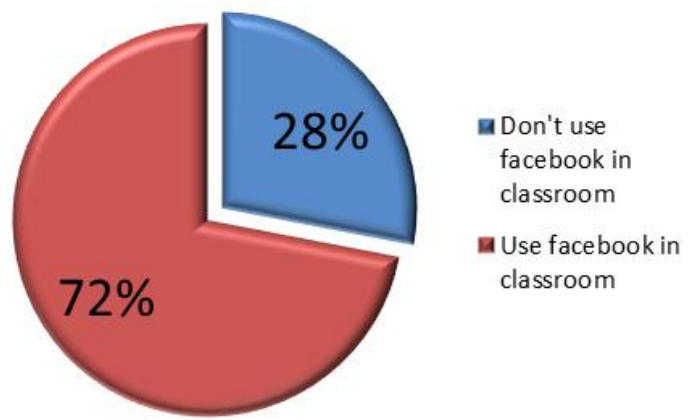

Fig 2: Facebook uses in classroom

Now it is very common question that while connecting in Facebook, how students can concentrate on their class. Consequently, our next survey emphasized on discovering the working efficiency of the people at the time of using Facebook. Hence we need to find out the people who use Facebook and work together. Among the 100 interviewee, $76 \%$ often get logged in into Facebook while working (Figure $3)$.

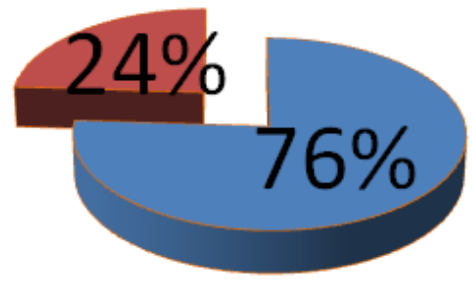

facebook+work

- Only face book

Fig 3: People who use Facebook while working

From these $76 \%$ people, only 5\% said that they can concentrate (Figure 4) into work while using Facebook. The other $95 \%$ feel disturbance to work. But it is not possible to give up Facebook by them and true to say people need more time to stay connected. Hence our working efficiency is greatly hampered and often reducing by social network.

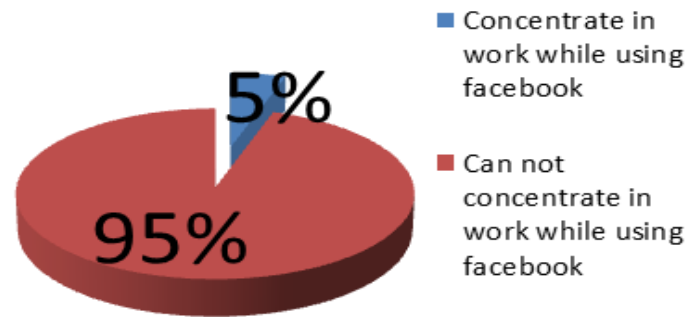

Fig 4: Only a few people can concentrate on work while using Facebook.

\subsection{Resultant Decision of Survey}

As the result of the survey it can be said that the current system is totally conflicting to our daily life and it consumes people's working performance. Hence it is crying need to develop a future social network system which can save our time to satisfy the need of the era.

\section{FUTURE SOCIAL NETWORK}

\subsection{System Design}

The future social network is based on some future technologies. Through collaborating BCI (Brain Computer Interfacing) and cloud computing a future social network can be designed.

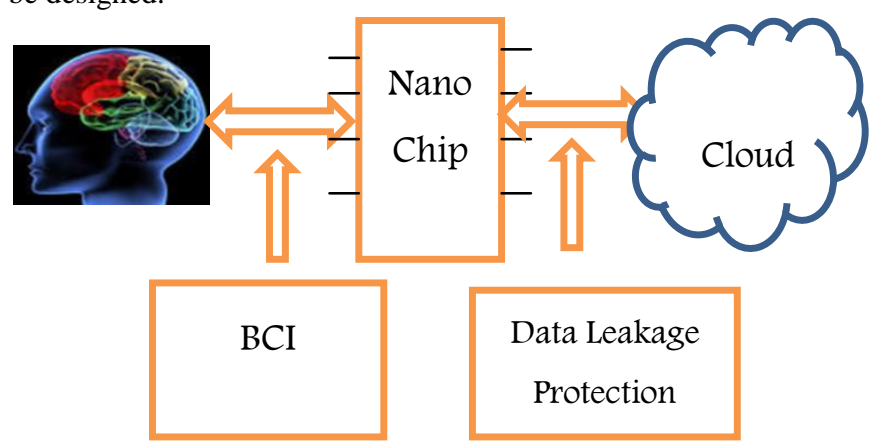

Fig 5: Proposed System design for future social network system.

In the system design (Figure 5) a Nano chip is connected with the brain by the BCI (brain computer interfacing) technology. It is actually planted into the brain connecting to neurons. The internal connections of the chip are photonic communication technology [4] based. The Nano chip can connect to the cloud and fetches data to provide to the neurons. While interacting to cloud, it is compulsory to ensure the safety of brain data. Brain data is ones saved memories, thinking and knowledge preserved into the brain. Nano chip contains all the programmatic commands which can act as a computer to browse social network. The whole process can run into the brain as a single process and don't hamper other processes of the brain.

\subsection{Brain Computer Interfacing}

A brain-computer interface (BCI) is a direct communication pathway between the brain and an external device (Figure 6) [8].

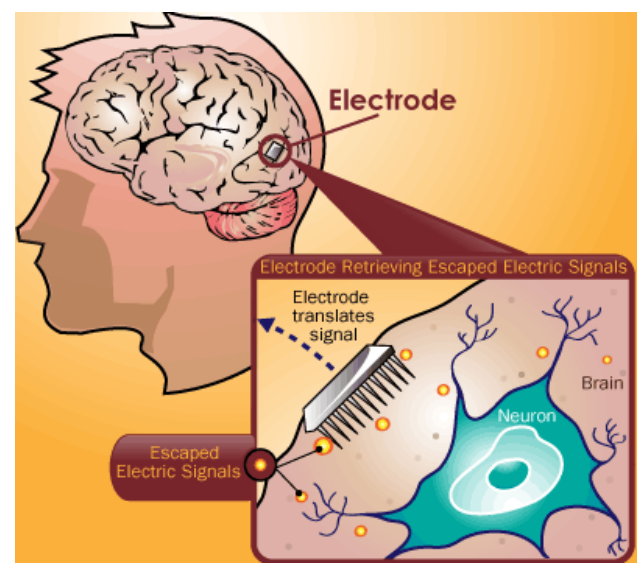

Fig 6: Brain computer interface.

Our brains are filled with neurons, individual nerve cells connected to one another by dendrites and axons. Every time we think, move, feel or remember something, our neurons are at work. That work is carried out by small electric signals. The signals are generated by differences in electric potential carried by ions on the membrane of each neuron. BCI can detect those signals, interpret what they mean and use them as 
command to the Nano chip. After having the proper command from brain, the chip communicates with cloud to browse social network.

\subsection{Cloud to Provide Resource}

Cloud computing is a synonym for distributed computing over a network and means the ability to run a program on many connected computers at the same time. It also refers to network based services which appear to be provided by real server hardware, which in fact served up by virtual hardware, simulated by software running on one or more real machines. For future social network, cloud can act as the resource of various data information to provide to the Nano chip. As the server of the social network will stay in the cloud, different Nano chips of different users will has to be cloud computing enabled.

\subsection{Combining Nano Technology and Photonic Communication Technology}

The very first challenge to design a microchip which can be planted into human brain is the size of the chip. The chip has to be as small as a neuron. The only hope is Nano technology. Then the next challenge is power consumption. The only source of power is the neurons which deliver a very small electrical signal. To ensure the best optimization of this power, best internal connections into the chip are needed. The upcoming photonic communication technology can satisfy that. Hence the best way is to combine the two technologies. Nano is to make the chip small and photonic for internal connections.

\subsection{Map Reduce for Cloud Server}

The proposed cloud server has to meet lot of complexities and load in future because the system gives the all-time using ability for the users. Hence it is needed to introduce a new method for massive data processing. Map Reduce [3] is a very popular parallel programming model for cloud computing platforms, and has become an effective method for processing massive data by using a cluster of computers. X-to-Map Reduce ( $\mathrm{X}$ is a program language) translator is a possible solution to help traditional programmers easily deploy an application to cloud systems through translating sequential codes to Map Reduce codes. A simple method [3] to translate Mat lab code to Map Reduce code is introduced from the following three aspects:

(1) Translate single Mat lab command to MapReduce code;

(2) Translate multiple Mat lab independent commands to MapReduce code and,

(3) Translate multiple Mat lab dependent commands to MapReduce code. We briefly introduce the flow chart (Figure 7) of M2M (Mat lab to MapReduce).

It consists of the following three stages:

(1) The first stage is a lexical analyzer, used to the token generation. The scanner begins the analysis of the Mat lab source code by reading the input, character by character, and grouping characters into meaningful words and symbols (tokens) defined by a grammar of regular expressions.

(2) The second stage is a syntax analyzer, used for parsing. Given a formal syntax specification (typically as a ContextFree Grammar (CFG)), the syntax analyzer reads tokens and groups them into units as specified by the productions of the CFG being used.
(3) The third stage is a semantic analyzer (translator), used for semantic parsing or analysis. It is working out the implications of the expression just validated and taking the appropriate action. Here, aiming at the Mat lab operations, a simple Math Operation Library based on MapReduce (MOLM) is built. When the translator processes a math command, it connects to the MOLM, gets the corresponding MapReduce code, and combines with a main function.

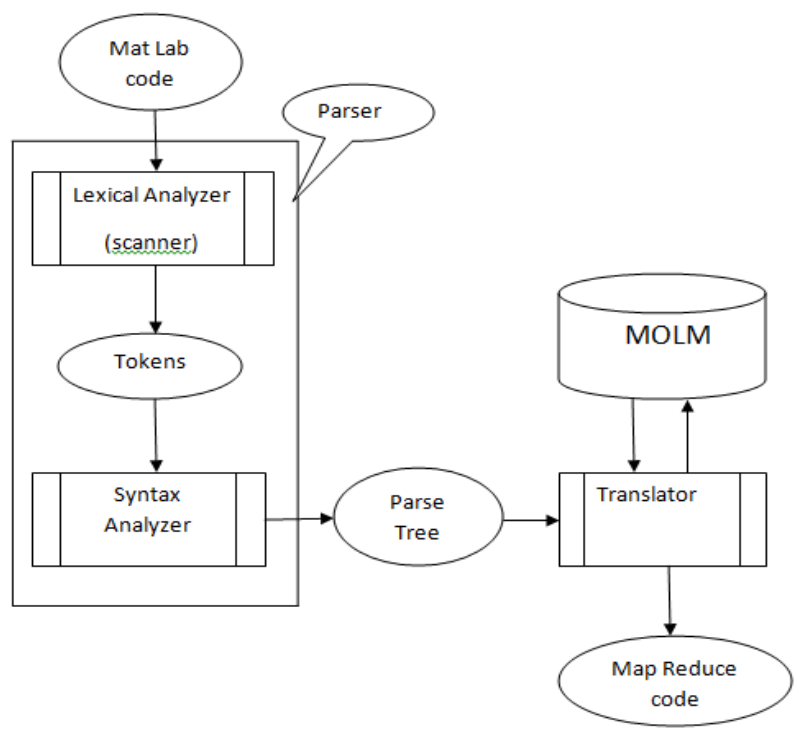

Fig 7: Flow chart of M2M.

\section{CONCLUSION}

In the current time social networks are part and parcel of daily life. Collaboration of BCI with cloud computing can save our working efficiency while using social network. The system acts as a single process in the brain and uses only a little portion for getting command. Moreover, the system does not require hands and hands are remaining free for other works. Thus people can use social network and do their daily work together without any efficiency loss. The challenge is data safety for brain. While connecting to the cloud a data leakage from brain may happen if we fail to give proper data protection. But the bad effects showed by survey results are not negligible. So the generation needs to switch to future technologies to overcome losing working efficiency by social networks.

\section{REFERENCES}

[1] Karl D. Stephan, Katina Michael, M. G. Michael, Laura Jacob, Emily P. Anesta, Vol. 100,May 13th, 2012. Social Implications of Technology: The Past, the Present, and the Future. Introducing the IEEE Society on Social Implications of Technology.

[2] Zhen Chen, Fuye Han, Junwei Cao, Xin Jiang, Shuo Chen, Vol 18,February 2013. Cloud Computing-Based Forensic Analysis for Collaborative Network. Tisinghua Science and Technology.

[3] Junbo Zhang, Dong Xiang, Tianrui Li, and Yi Pan, Vol 18, February 2013.M2M: A Simple Matlab-toMapReduce Translator for Cloud Computing. Tisinghua Science and Technology.

[4] Robert F. Leheny, Vol. 100, May 13th, 2012. Molecular Engineering to Computer Science: The Role of Photonics 
in the Convergence of Communications and Computing. Proceedings of the IEEE.

[5] Yen-Kuangchen, An-Yeuwu, Magdya. Bayoumi, Farinazkoushanfar, Vol. 3, March 2013. Editorial LowPower, Intelligent, and Secure Solutions for Realization of Internet of Things. IEEE Journal on Emerging and Selected Topics in Circuits and Systems.

[6] Federico Maggi, Stefano Zanero, 2013. Is the future Web more insecure? Distractions and solutions of new-old security issues and measures. WEI.

[7] K. D. Stephan, 2006. Notes for a history of the IEEE society on social implications of technology. IEEE Technol. Soc. Mag.

[8] Ed Grabianowski, 2007. How Brain-computer Interfaces Work. How Stuff Works [online]. Available: http://computer.howstuffworks.com/brain-computerinterface.htm.

[9] Bing Liu, April 22, 2012. Sentiment Analysis and Opinion Mining. Claypool Publishers.
[10] Zhu Zhang, Xin Li, 2010.43rd Hawaii International Conference on System Sciences. Controversy is Marketing: Mining Sentiments in Social Media.

[11] G.Vinodhini, RM.Chandrasekaran,June 2012. Sentiment Analysis and Opinion Mining: A Survey. International Journal of Advanced Research in Computer Science and Software Engineering.

[12] B. He, W. Fang, Q. Luo, N. K. Govindaraju, and T. Wang, 2008. Mars: A mapreduce framework on graphics processors. Proc. of the 17th International Conference on Parallel Architectures and Compilation Techniques, New York.

[13] T. Gunarathne, B. Zhang, T.-L. Wu, and J. Qiu, 2011 Portableparallel programming on cloud and hpc: Scientificapplications of twister4azure, Utility and Cloud Computing (UCC) Fourth IEEE Int. Conf.

[14] K. Kc and K. Anyanwu,2010. Scheduling hadoop jobs to meet deadlines, Cloud Computing Technology and Science(CloudCom) IEEE Second Int. Conf. 